\title{
BMJ Open Vascular biomarkers to predict response to exercise in Alzheimer's disease: the study protocol
}

\author{
Danni Li, ${ }^{1}$ Robin Thomas, ${ }^{1}$ Michael Y Tsai, ${ }^{1}$ Ling Li, ${ }^{2}$ David M Vock, ${ }^{3}$ \\ Susan Greimel, ${ }^{4}$ Fang $\mathrm{Yu}^{4}$
}

To cite: Li D, Thomas $R$ Tsai MY, et al. Vascular biomarkers to predict response to exercise in Alzheimer's disease: the study protocol. BMJ Open 2016:6:e011054. doi:10.1136/bmjopen-2016011054

- Prepublication history for this paper is available online. To view these files please visit the journal online (http://dx.doi.org/10.1136/ bmjopen-2016-011054).

Received 6 January 2016 Revised 10 October 2016 Accepted 13 October 2016

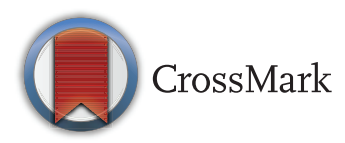

\footnotetext{
${ }^{1}$ Department of Laboratory Medicine and Pathology, University of Minnesota, Twin Cities, Minneapolis, Minnesota, USA

${ }^{2}$ Department of Experimental and Clinical Pharmacology, College of Pharmacy, University of Minnesota, Twin Cities, Minneapolis, Minnesota, USA

${ }^{3}$ Division of Biostatistics, School of Public Health, University of Minnesota, Twin Cities, Minneapolis, Minnesota, USA

${ }^{4}$ School of Nursing, University of Minnesota, Twin Cities, Minneapolis, Minnesota, USA
}

Correspondence to Dr Danni Li; dannili@umn.edu

\section{ABSTRACT}

Introduction: Exercise interventions are a promising treatment for improving cognition in persons with Alzheimer's disease. This is similar to Alzheimer's disease pharmacotherapies in which only $18-48 \%$ of treated patients demonstrate improvement in cognition. Aerobic exercise interventions positively affect brain structure and function through biologically sound pathways. However, an under-studied mechanism of aerobic exercise's effects is n-3 fatty acids in plasma. The objective of this pilot study is to inform a future large-scale study to develop n-3 fatty acids-based prediction of cognitive responses to aerobic exercise treatment in Alzheimer's disease.

Methods and analysis: This study will recruit and follow a cohort of 25 subjects enrolled in the FIT-AD Trial, an ongoing randomised controlled trial that investigates the effects of a 6 -month moderateintensity cycling intervention on cognition and hippocampal volume in older adults with mild to moderate Alzheimer's disease over a year. This study will collect blood from subjects at baseline and at 3 and 6 months to assay vascular biomarkers (ie, plasma fatty acids). Global cognition as measured by the Alzheimer's Disease Assessment Scale-Cognition (ADAS-Cog) at baseline, 3, 6, 9 and 12 months will be used as the main outcome. A multiple linear-regression model will be used with 12-month change in cognition as the outcome and baseline measure of n-3 fatty acids or changes in the ratio of $n-3$ to $n-6$ fatty-acid levels in plasma at 3 and/or 6 months, randomised treatment group, and their interaction as predictors.

Ethics and dissemination: We have obtained Institutional Review Board approval for our study. We obtain consent or assent/surrogate consent from all subjects depending on their consenting capacity assessment. Data of this study are/will be stored in the Research Electronic Data Capture (REDCap). We plan to present and publish our study findings through presentations and manuscripts.

Trial registration number: NCT01954550.

\section{BACKGROUND}

Alzheimer's disease (AD) presents a public health priority worldwide. ${ }^{2}{ }^{2}$ In the USA

\section{Strengths and limitations of this study}

- Our pilot study is embedded within a well designed FIT-AD Trial.

- Specific aims are supported by strong scientific premises.

- Results may be limited to aerobic exercise.

- Results may be limited to older adults with mild to moderate Alzheimer's disease only.

alone, there were an estimated 5.3 million people with $\mathrm{AD}$, with an estimated yearly cost of $\$ 226$ billion, ${ }^{3}$ which is projected to increase to $\$ 511$ billion in 2040 if effective therapies are not developed to prevent, slow or stop this devastating disease. ${ }^{3}$ An effective treatment will need to alter $\mathrm{AD}$ pathological changes (ie, $\beta$ amyloid plaques and neurofibrillary tangles) in the brain. However, two decades of drug clinical trials targeting those pathologies have failed at $99.6 \%$ rate, ${ }^{4}$ suggesting the pressing need to investigate other mechanisms such as vascular biomarkers of AD. Plasma n-3 fatty acids are one such potential mechanism. Lower levels of n-3 fatty acids in red blood cells, especially docosahexaenoic acid (DHA), were associated with cognitive impairment even in persons free of clinical dementia. ${ }^{5}$ Levels of plasma n-3 fatty acids were lower in $\mathrm{AD}^{6}$ and were associated with lower Mini Mental Status Exam (MMSE) scores in individuals with $\mathrm{AD}^{7}$ On the molecular level, exercise and fatty acids share some similar effects on cognition. Both act on dopaminergic pathways, ${ }^{89}$ and influence the expression of brainderived neurotrophic factor, which promote synaptic plasticity, cell proliferation, and cell survival in humans and rodents. ${ }^{10} 11$ Because of the shared neurobiological and physiological effects, several human and animal studies have speculated about the benefits of higher, endogenous levels of n-3 fatty acids in plasma in contribution to the pro- 
cognitive effects of exercise. ${ }^{12}{ }^{13}$ Recently, Chytrova et $a l^{14}$ showed that exercise increased expression of synaptic plasticity and membrane structure proteins in adult male Sprague-Dawley rats supplemented with n-3 fatty acids more than in rats without the supplementation. Although the findings from Chytrova et al was based on n-3 fatty acid supplementation, it suggests that, in individuals with $\mathrm{AD}$, higher endogenous levels of n-3 fatty acids levels may be associated with better cognitive response to exercise treatment.

Furthermore, interventions such as aerobic exercise that influence n-3 fatty acid levels in plasma might be effective for improving cognition, delaying functional decline, and reducing the prevalence and impact of behavioural and psychological symptoms of dementia. ${ }^{15-17}$ Aerobic exercise has been postulated to affect cognition through several direct mechanisms that have been widely tested, including improving cerebral blood flow, ${ }^{18}$ stimulating neurogenesis, ${ }^{19}$ increasing brain volume, ${ }^{20}$ and boosting functional connectivity and plasticity. ${ }^{21}$ An untapped mechanism is exercise's indirect effects on vascular biomarkers of $\mathrm{AD}$ such as $\mathrm{n}-3$ fatty acids. Exercise increases the relative levels of $n-3$ fatty acids (ie, the ratio of n-3 to $n-6$ fatty acids) in plasma. Andersson et al showed that the ratio of n-3 to n- 6 fatty acids was significantly higher in leg muscles of exercise trained healthy men than untrained ones, which was presumably due to preferential oxidation of n-6 fatty acids over n-3 fatty acids and increase in activities of lipid-metabolising enzymes. ${ }^{22}{ }^{23}$ These exercise-induced increases in the ratio of $n-3$ to $n-6$ fatty acids in skeletal muscle have also been observed in plasma. ${ }^{24}$ Plasma n-3 fatty acids have anti-oxidative and anti-inflammatory properties that contribute to their cerebrovascular protective effects on cognition in $\mathrm{AD} .^{25}{ }^{26}$ Taken together, an exercise-induced increase in the ratio of $n-3$ to $n-6$ fatty-acid levels in plasma may serve as an early biomarker (surrogate endpoint) for long-term cognitive responses to exercise interventions.

\section{Study aims}

The objective of this pilot cohort study is to inform a future large-scale study to develop n-3 fatty acids based prediction of cognitive responses to exercise in $\mathrm{AD}$. The specific aims and hypotheses of the study are:

\section{Aim 1: Identify vascular biomarkers at baseline that predict cognitive responses to exercise in $\mathrm{AD}$}

Hypothesis 1: Higher levels of plasma n-3 fatty acids at baseline are associated with favourable changes in cognition at 12 months.

\section{Aim 2: Evaluate changes in vascular biomarkers as surrogate endpoints for early measure of cognitive responses to exercise in $A D$}

Hypothesis 2: Larger increases in the ratio of $\mathrm{n}-3$ to $\mathrm{n}-6$ fatty-acid levels in plasma at 3 and/or 6 months are associated with favourable changes in cognition at 12 months.
METHODS

\section{Design}

We will use a cohort design to recruit 25 subjects enrolled in the FIT-AD Trial from both the cycling intervention and sham exercise groups. The FIT-AD Trial is a pilot randomised controlled trial that investigates the effects of a 6-month, individualised, moderate-intensity cycling intervention on cognition and hippocampal volume in community-dwelling older adults with mild to moderate AD (defined by MMSE score of 15-16). ${ }^{27}$ The trial, currently ongoing at the University of Minnesota (UMN), randomises 90 subjects to the 6-month moderate-intensity cycling intervention or sham exercise using a 2:1 allocation ratio and follows them for an additional 6 months after the exercise intervention from 1 August 2013 to 30 April 2019 and each recruitment year ends on 30 April. The FIT-AD trial began recruitment in March 2014 and enrolled its first subject on 3 June 2014. The FIT-AD trial met its enrolment goals of 24 and 22 subjects for years 1 and 2, respectively, and began its year 3 enrolments on 1 May 2016 and had enrolled 2 of the 22 subjects by 30 May 2016. The details of the exercise intervention have been published elsewhere. ${ }^{27}$ Stretching exercise is used in the FIT-AD Trial as sham exercise with intensity not exceeding $20 \%$ of the heart rate reserve or Rating of Perceived Exertion of 8, and includes primarily seated movements and static stretches. ${ }^{27}$ In the FIT-AD Trial, global cognition is measured by the AD Assessment Scale-Cognition (ADAS-Cog) at baseline, 3, 6, 9 and 12 months and shared with this study. In this study, we will collect fasting blood samples from subjects at baseline and at 3 and 6 months and measure n-3 and n- 6 plasma fatty-acid levels. Study subjects' apolipoprotein E (ApoE) genotype will also be determined for two reasons: the ApoE 4 allele is a well established risk factor for $\mathrm{AD},{ }^{28}$ and $\mathrm{ApoE}$ genotype has been shown to affect lipid metabolism. ${ }^{29}$

\section{Settings}

Blood collection will be performed at the UMN Clinical and Translational Science Institute (CTSI) by experience phlebotomists. A graduate research assistant will provide subjects with free transportation for blood collection using a rented university vehicle with liability coverage. The samples will be stored in a $-70^{\circ} \mathrm{C}$ freezer in the Drug Analysis Laboratory (DAL) at the UMN Medical Center and processed by the laboratory technician (RT) in the first author's laboratory. Plasma fatty acids analysis will be performed in MT's laboratory at the UMN Department of Laboratory Medicine and Pathology (LMP). DNA extraction and ApoE genotype analysis will be performed in the Advanced Research and Diagnostic Laboratory (ARDL) at the UMN Department of LMP. All personnel involved in blood collection, processing, and biomarker assessment are blinded to subjects' FIT-AD Trial treatment group assignment and cognitive outcomes. 


\section{Study population}

The study population is community-dwelling older adults with mild to moderate AD. Subjects meeting the FIT-AD Trial eligibility criteria ${ }^{27}$ will need to meet two additional requirements to be enrolled in this study: agree to donate a total of $30 \mathrm{~mL}$ of blood $(10 \mathrm{~mL}$ at each blood collection); and fast for at least more than 8 hours (without any food or drink intake other than water and prescribed medicines) before blood collection. Venous blood collections by trained phlebotomists are considered low risk for subjects.

\section{Ethics, consent and permissions}

Twenty-five subjects from the 66 subjects anticipated to be enrolled in the FIT-AD Trial during years 2-4 will be recruited into this study. The FIT-AD Trial project manager (SG) will also serve as project manager for this study. The project manager will recruit subjects to the FIT-AD Trial and this cohort study, and will obtain consent for the FIT-AD Trial and this study at the same time. We will only enrol subjects who have been successfully enrolled in the FIT-AD Trial and consented to be in this study. Specifically, the project manager will approach all consecutive enrolled subjects in the FIT-AD Trial before they are randomised, and ask their interest in participating in this study. If there is interest, the project manager will provide them with a consent form and obtain informed consent or assent/surrogate consent from all subjects depending on their consenting capacity assessment. Surrogates will be identified in the descending order of power of attorney, spouse, adult children and significant others. Subjects' or their surrogates' understanding of the study and capacity of decision-making will be checked using the 'Assessment of Subject's Understanding of the Study' form with 10 questions specifically designed for this study. Individuals with $\geq 80 \%$ correct answers after one trial can sign their own consent. Those with $<80 \%$ correct answers after one trial will need a family member to sign the surrogate consent with the participant to sign the assent form. The project manager will obtain consent at baseline and re-assess consent prior to each subsequent data collection, ${ }^{27}$ and submit any subsequent changes or amendments to the protocol to the Institutional Review Board (IRB) for approval. This study is approved by the UMN's IRB: \# 1508M77566. The recruitment of this study started in December 2015.

\section{Sample size}

The emphasis of this pilot study is to provide preliminary results to estimate effect sizes for power calculation in a future large-scale study. Using a conservative retention rate of $85 \%$ (year 1 retention rate in the FIT-AD Trial was $95 \%$ as of April 2015), an anticipated 30 enrolees will produce a target sample size of 25 .

\section{Retention}

The FIT-AD Trial uses 15 retention strategies. ${ }^{27}$ Five additional strategies will be used for subject retention in this study: (1) conducting phone calls after blood collection to address concerns and ensure future participation; (2) sending birthday and thank-you cards to establish rapport; (3) utilising flexible scheduling of blood collection; (4) providing transportation to blood collection; and (5) compensating subjects.

\section{Blood collection and processing}

The project manager will schedule the blood collections after but within the same week of the corresponding cognitive assessments (at baseline and at 3 and 6 months). The day before the blood collection, the project manager will remind subjects and their caregivers of their appointments by phone, and ask subjects to fast, and their caregivers to ensure that subjects fast. Subjects will be allowed to have water and medications but should refrain from food for at least 8 hours prior to their appointment time (eg, starting at midnight). Subjects are encouraged to drink water before blood collection to ensure an uneventful blood collection. Subjects with diabetes will be instructed to check blood glucose levels frequently during fasting and stop fasting immediately if blood glucose levels exceed $300 \mathrm{mg} / \mathrm{dL}$ or drop lower than $70 \mathrm{mg} / \mathrm{dL}$. In addition, the project manager will schedule a blood collection appointment for subjects with diabetes as early as possible in the morning to prevent a longer fast. Snacks will be provided to all subjects (regardless of whether they have diabetes or not) immediately after blood collection to prevent hypoglycaemia.

On the day of blood collection, the graduate research assistant will ask subjects and their caregivers about food intake during the previous 8 hours. If a subject did not fast, the graduate research assistant will notify the project manager who will reschedule the blood draw. If a subject has fasted, the graduate research assistant will transport the subject to the UMN CTSI. A phlebotomist will collect a total of $10 \mathrm{~mL}$ of blood into a $6 \mathrm{~mL}$ plasma (EDTA) tube and a $4 \mathrm{~mL}$ serum tube. These tubes will be labelled with the FIT-AD Trial patient identification number. After collection, phlebotomists will gently mix the tubes and store the tubes on ice. The graduate research assistant will notify the laboratory technician RT from DL's laboratory to pick up the specimens at the CTSI within an hour.

The plasma and serum tubes will be gently mixed and centrifuged in $4^{\circ} \mathrm{C}$ using a temperature-controlled centrifuge (ie, Sorvall Heraeus Multifuge $3 \mathrm{~S}-\mathrm{R}$ ) with a Swing out Rotor (ie, \#75 006445 ) at $3000 \mathrm{~g}$ for $10 \mathrm{~min}$ in the DAL analysis laboratory. The tubes will be removed from the centrifuge immediately after completion. From the plasma tube, nine plasma aliquots (one of $500 \mu \mathrm{L}$, one of $50 \mu \mathrm{L}$ and seven of $250 \mu \mathrm{L}$ ) will be made; from the serum tube, eight serum aliquots of $250 \mu \mathrm{L}$ will be made; red blood cells from the plasma tube will be transferred into a $5 \mathrm{~mL}$ DNA phial. The aliquoted samples (ie, plasma, serum and packed cells separated from the plasma) will be stored in the $-70^{\circ} \mathrm{C}$ freezer in the DAL. 
Each subject can determine the use of his or her samples post study. If a subject indicates he or she would prefer the specimens not be stored for future use, these specimens will be discarded on completion of this study. If a subject indicates the specimens can be used for future studies, the specimens will continue to be stored in the $-70^{\circ} \mathrm{C}$ freezer in the DAL.

\section{Biomarker assessment}

Biomarker analyses will be performed when all subjects' blood samples are collected. Samples will be analysed in a single batch to avoid batch effects. Plasma fatty acids will be analysed using a method based on a Hewlett Packard 5890 gas chromatograph (Hewlett Packard, Wilmington, Delaware, USA) ${ }^{30}$ that measures 28 phospholipid fatty acids, including 3 n-3 fatty acids $(\alpha$-linolenic acid (ALA), eicosapentaenoic acid (EPA) and DHA) and 2 n-6 fatty acids (linoleic acid (LA) and arachidonic acid (AA)). This method has the following coefficient of variations (CV) obtained by 20 replicates in consultant MT's laboratory: ALA, 2.4\%; EPA, 3.3\%; DHA, 2.7\%; LA, 2.6\%; and AA, 2.4\%. DNA will be extracted from white cell counts stored at $-70^{\circ} \mathrm{C}$ using Puregene reagents (Qiagen, Germantown, Maryland, USA) and ApoE genotype will be determined using Taqman SNP Genotyping assays for rs429358 and rs7412 (Life Technologies, Carlsbad, California, USA).

\section{Data management}

The principle investigator (PI) of the FIT-AD Trial (FY) will manage all the data collected by FIT-AD Trial data and conduct data audits to ensure accuracy and completeness of the FIT-AD Trial data. The PI of this study DL will manage all the biomarker data for this study. Quality control samples will be measured in replicates during biomarker analyses to ensure accuracy and precision of the biomarker assessment. All the biomarker data along with the data collected as part of the FIT-AD trial will be stored in the Research Electronic Data Capture (REDCap). REDCap is a secure web interface with data checks during data entry and uploading to ensure data quality, and housed on secure severs operated by the UMN Academic Health Center's Information Systems.

\section{Statistical analysis}

Aim 1: Identify vascular biomarkers at baseline that predict cognitive responses to exercise in $A D$

To qualitatively address Aim 1, the change in cognition over 12 months (eg, mean, median, minimum and maximum) will be calculated between the two randomised treatment groups and between different categories of baseline measures of n-3 fatty acids (eg, different quantiles of DHA). To estimate an effect size for the interaction of baseline n-3 fatty acids and exercisetreatment response, a multiple linear-regression model will be used with 12-month change in cognition as the outcome and baseline measure of n-3 fatty acids, randomised treatment group, and their interaction as predictors. Separate models will be fit for each measure of n-3 fatty acids (ie, ALA, EPA and DHA). Other demographic and clinical characteristics, such as age, education, gender, ApoE genotype, and exercise adherence will be adjusted for in these models. Given the moderate sample size, it is likely that only one or two other covariates could be included in the model before the model estimates become unstable.

\section{Aim 2: Evaluate changes in vascular biomarkers as surrogate endpoints for early measure of cognitive responses to exercise in $A D$}

To address Aim 2, correlations, both Pearson and Spearman rank, will be computed between the 3-month or 6-month changes in the ratio of n-3 to n-6 fatty-acid levels and the change in cognition at 12 months measured by ADAS-Cog. Several definitions of the ratio of n-3 to n-6 fatty acids will be considered, which include (EPA+DHA)/AA and DHA/AA. ${ }^{31}{ }^{32}$ In addition, how best to quantify the change in this ratio will be explored using the difference between baseline and 3 or 6 months, ratio between baseline and 3 or 6 months, etc. If the change in the ratio is strongly skewed, logtransforming those measures may be considered. For biomarkers in which the change in biomarker level is moderately to strongly correlated to the change in cognition in the univariate analyses (Pearson $r>0.4$ ), the association between the variable(s) and cognition will be examined using a multiple linear-regression model. The covariates for inclusion may be age, education, gender, ApoE genotype, baseline values of the biomarker, and randomised treatment group. It is expected that the change in the ratio between baseline and 6 months will more likely be associated with change in cognition at 12 months than will the change in the ratio between baseline and 3 months.

\section{Additional analytical considerations}

Because this is a pilot study designed to estimate effect sizes for a future large-scale study, no formal hypothesis testing will be conducted. Because the results will only be used for generating hypotheses, no adjustment will be made for the comparison of multiple n-3 or n-6 fatty acids. The primary end point for both aims will be the change in cognition measured at 12 months, but the change measured at other time points (eg, 6 and 9 months) will be considered as part of exploratory analyses as well.

\section{Dissemination}

Findings from the study will be disseminated through publications and presentations. Authorship will follow the established publication guidelines as the International Committee of Medical Journal Editors. We do not intend to use professional writers. 


\section{DISCUSSION}

A few factors affected how we designed this study. First, n-3 fatty acid supplements, including fish oil, are commonly taken in patients with $\mathrm{AD}$, which increase plasma n-3 fatty acids. Because the FIT-AD Trial conducts monthly monitoring of medication changes in subjects, we will use that information to exclude from data analysis those subjects who start, stop or change the frequency of taking these supplements during the study. This should not affect Aim 1, which evaluates baseline biomarkers. This may affect Aim 2, which evaluates changes in biomarkers. However, excluding subjects should not impact the objective of this pilot study, which is to estimate effect sizes for power calculation in the future large-scale study.

Second, it is well known that pre-analytical variables in sample collection and processing may affect concentrations of fatty acids in plasma. ${ }^{33}$ Therefore, when we designed our protocols to collect and process blood samples, we made sure our protocols are compatible with stability of fatty acids in plasma, such as placing the blood samples on ice immediately after the collection, and centrifuging the specimens at $4^{\circ} \mathrm{C}$, and storing the samples long term in a freezer at $-70^{\circ} \mathrm{C}$.

Third, although cognitive data will be collected at baseline, 3, 6, 9 and 12 months as part of the FIT-AD Trial, and shared with this study, blood samples in this study will be collected only at baseline, 3 and 6 months, not at 9 and 12 months. This is because changes in vascular biomarkers (ie, plasma n-3 fatty acids) during the follow-up are not within the scope of this study. The goals are to determine whether vascular biomarkers at baseline or changes during the exercise predict improved or stabilised ADAS-Cog at 12 months.

Finally, sham exercise will be used as a control to ensure that the predictive biomarkers identified are indeed associated with exercise treatment, rather than representative of prognostic biomarkers, since prognostic biomarkers may indicate good prognosis and slower disease progression regardless of whether the patient received exercise intervention or not. We will not try to balance the subjects in terms of treatment group (cycling intervention vs sham exercise groups).

There are three limitations to our study. First, we chose global cognition measured by the ADAS-Cog as the outcome in this study because the ADAS-Cog is the FDA-approved cognitive measure for drug RCTs and will allow us to compare our results with other published studies. However, this global measure may not detect cognitive changes in specific cognitive domains that have differential responses to aerobic exercise intervention. The FIT-AD Trial will collect cognitive measures of specific domains, including attention, processing speed, executive function, memory and language, ${ }^{27}$ which may be used in hypothesis-generating analyses in this study. Second, results from the FIT-AD Trial may be limited to aerobic exercise, and may not reflect other forms of exercise (eg, anaerobic) or interventions with different intensity or duration. Finally, results from the study will be limited to older adults with mild to moderate $\mathrm{AD}$ only and are not generalisable to individuals with severe $\mathrm{AD}$ or early-onset, familial AD.

This pilot cohort study will generate preliminary results needed for power calculation in a future large-scale study to develop a biomarker-based prediction of cognitive responses to exercise in $\mathrm{AD}$. This pilot study will constitute a first step towards future studies to provide important knowledge on the degree to which plasma n-3 fatty acids are associated with cognitive responses to exercise treatment among patients with $\mathrm{AD}$. If plasma n-3 fatty acids were found to predict cognitive responses of aerobic exercise interventions in $\mathrm{AD}, \mathrm{n}-3$ supplementation may be recommended to enhance the pro-cognitive effects of exercise, further slowing down cognitive decline in patients with $\mathrm{AD}$. Cognitive decline and associated physical function impairment in $\mathrm{AD}$ is the leading cause of nursing home placement. ${ }^{34} 35$ Interventions directed to slowing down cognitive and other related functional impairment, and hence nursing home placement, will not only improve quality of life of patients with $\mathrm{AD}$, but also save billions in formal care. ${ }^{36}$ Personalised treatment has revolutionised therapies for cancer. ${ }^{37}$ There is good evidence to support the value of personalised interventions to address behavioural and psychological symptoms in people with dementia and to improve their quality of life. ${ }^{38} 39$ Our pilot study will generate the knowledge to inform future studies, which will be critical for developing effective, personalised exercise treatments in $\mathrm{AD}$, which will likely maximise resources, reduce healthcare costs, and improve treatment efficacy and quality of life for patients with $\mathrm{AD}$.

\section{STUDY STATUS}

The status of the cohort study at the time of manuscript submission is open for enrolment. We started enrolment in January 2016, and expect enrolment accrual to complete in November 2017.

Contributors DL designed and supervises all aspects of the study, and drafted the manuscript. RT contributed to the draft of the manuscript. MT supervises plasma fatty acids analysis and contributed to the draft of the manuscript. LL contributed to the draft of the manuscript and provided feedback to the design of the study. SG contributed to the draft of the manuscript. DV contributed to the draft of the manuscript, provided feedback to the design of the study, and designed the statistical analysis plan. FA contributed to the draft of the manuscript and provided significant feedback to the design of the study. All authors read and approved the final manuscript.

Funding This study is funded by the Alzheimer's Association (NIGR-15-362392, 1 December 2015 to 30 November 2017).

Competing interests None declared.

Ethics approval This study was approved by the UMN's IRB: \# 1508M77566.

Provenance and peer review Not commissioned; externally peer reviewed.

Open Access This is an Open Access article distributed in accordance with the Creative Commons Attribution Non Commercial (CC BY-NC 4.0) license, which permits others to distribute, remix, adapt, build upon this work noncommercially, and license their derivative works on different terms, provided the original work is properly cited and the use is non-commercial. See: http:// creativecommons.org/licenses/by-nc/4.0/ 


\section{REFERENCES}

1. Wimo A, Winblad B, Jönsson L. The worldwide societal costs of dementia: estimates for 2009. Alzheimers Dement 2010;6:98-103.

2. Prince $\mathrm{M}$, Bryce R, Albanese $\mathrm{E}$, et al. The global prevalence of dementia: a systematic review and metaanalysis. Alzheimers Dement 2013:9:63-75.

3. Association AS. 2015 Alzheimer's disease facts and figures. Alzheimers Dement 2015;11:332-84.

4. Cummings JL, Morstorf T, Zhong K. Alzheimer's disease drug-development pipeline: few candidates, frequent failures. Alzheimers Res Ther 2014;6:37-44.

5. Tan ZS, Harris WS, Beiser AS, et al. Red blood cell omega-3 fatty acid levels and markers of accelerated brain aging. Neurology 2012;78:658-64.

6. Conquer JA, Tierney MC, Zecevic J, et al. Fatty acid analysis of blood plasma of patients with Alzheimer's disease, other types of dementia, and cognitive impairment. Lipids 2000;35:1305-12.

7. Wang W, Shinto L, Connor WE, et al. Nutritional biomarkers in Alzheimer's disease: the association between carotenoids, $\mathrm{n}-3$ fatty acids, and dementia severity. J Alzheimers Dis 2008;13:31-8.

8. Davis PF, Ozias MK, Carlson SE, et al. Dopamine receptor alterations in female rats with diet-induced decreased brain docosahexaenoic acid (DHA): interactions with reproductive status. Nutr Neurosci 2010;13:161-9.

9. Pothakos K, Kurz MJ, Lau YS. Restorative effect of endurance exercise on behavioral deficits in the chronic mouse model of Parkinson's disease with severe neurodegeneration. BMC Neurosci 2009;10:6.

10. Erickson KI, Voss MW, Prakash RS, et al. Exercise training increases size of hippocampus and improves memory. Proc Nat Acad Sci USA 2011;108:3017-22.

11. Wu A, Ying Z, Gomez-Pinilla F. Docosahexaenoic acid dietary supplementation enhances the effects of exercise on synaptic plasticity and cognition. Neuroscience 2008;155:751-9.

12. Gómez-Pinilla F, Feng $C$. Molecular mechanisms for the ability of exercise supporting cognitive abilities and counteracting neurologica disorders. In: Boecker H, Hillman C, Scheef L, eds. Functional Neuroimaging in Exercise Sport Sciences. Berlin: Springer, 2012:25-43.

13. Gomez-Pinilla F. The combined effects of exercise and foods in preventing neurological and cognitive disorders. Prev Med 2011;52 (Suppl 1):S75-80.

14. Chytrova G, Ying Z, Gomez-Pinilla F. Exercise contributes to the effects of DHA dietary supplementation by acting on membrane-related synaptic systems. Brain Res 2010;1341:32-40.

15. Heyn P, Abreu BC, Ottenbacher KJ. The effects of exercise training on elderly persons with cognitive impairment and dementia: a meta-analysis. Arch Phys Med Rehabil 2004;85:1694-704.

16. Toots $\mathrm{A}$, Littbrand $\mathrm{H}$, Lindelöf $\mathrm{N}$, et al. Effects of a high-intensity functional exercise program on dependence in activities of daily living and balance in older adults with dementia. J Am Geriatr Soc 2016;64:55-64.

17. Smith PJ, Blumenthal JA, Hoffman BM, et al. Aerobic exercise and neurocognitive performance: a meta-analytic review of randomized controlled trials. Psychosom Med 2010;72:239-52.

18. Rhyu IJ, Bytheway JA, Kohler SJ, et al. Effects of aerobic exercise training on cognitive function and cortical vascularity in monkeys. Neuroscience 2010;167:1239-48.

19. Hu YS, Xu P, Pigino G, et al. Complex environment experience rescues impaired neurogenesis, enhances synaptic plasticity, and attenuates neuropathology in familial Alzheimer's disease-linked APPswe/PS1 1 E9 mice. FASEB J 2010;24:1667-81.
20. Colcombe SJ, Erickson KI, Scalf PE, et al. Aerobic exercise training increases brain volume in aging humans. J Gerontol A Biol Sci Med Sci 2006;61:1166-70.

21. Voss MW, Prakash RS, Erickson KI, et al. Plasticity of brain networks in a randomized intervention trial of exercise training in older adults. Front Aging Neurosci 2010;2:1-12.

22. Andersson A, Sjödin A, Olsson R, et al. Effects of physical exercise on phospholipid fatty acid composition in skeletal muscle. $A m$ J Physiol 1998;274:E432-E38.

23. Andersson A, Sjödin A, Hedman A, et al. Fatty acid profile of skeletal muscle phospholipids in trained and untrained young men. Am J Physiol Endocrinol Metab 2000;279:E744-E51.

24. Mougios V, Kotzamanidis C, Koutsari C, et al. Exercise-induced changes in the concentration of individual fatty acids and triacylglycerols of human plasma. Metab Clin Exp 1995;44: 681-8.

25. Calon F, Cole $\mathrm{G}$. Neuroprotective action of omega-3 polyunsaturated fatty acids against neurodegenerative diseases: evidence from animal studies. Prostaglandins Leukot Essent Fatty Acids 2007;77:287-93.

26. Kalmijn S, Feskens EJ, Launer LJ, et al. Polyunsaturated fatty acids, antioxidants, and cognitive function in very old men. Am J Epidemiol 1997;145:33-41.

27. Yu F, Bronas UG, Konety S, et al. Effects of aerobic exercise on cognition and hippocampal volume in Alzheimer's disease: study protocol of a randomized controlled trial (The FIT-AD trial). Trials 2014;15:394.

28. Corder EH, Saunders AM, Strittmatter WJ, et al. Gene dose of apolipoprotein $\mathrm{E}$ type 4 allele and the risk of Alzheimer's disease in late onset families. Science 1993;261:921-3.

29. Mahley RW, Rall SC Jr. Apolipoprotein E: far more than a lipid transport protein. Annu Rev Genomics Hum Genet 2000;1:507-37.

30. Cao J, Schwichtenberg KA, Hanson NQ, et al. Incorporation and clearance of omega-3 fatty acids in erythrocyte membranes and plasma phospholipids. Clin Chem 2006;52:2265-72.

31. Beydoun MA, Kaufman JS, Satia JA, et al. Plasma n-3 fatty acids and the risk of cognitive decline in older adults: the Atherosclerosis Risk in Communities Study. Am J Clin Nutr 2007;85:1103-11.

32. Leckie RL, Manuck SB, Bhattacharjee N, et al. Omega-3 fatty acids moderate effects of physical activity on cognitive function. Neuropsychologia 2014;59:103-11.

33. Yin $\mathrm{P}$, Peter A, Franken $\mathrm{H}$, et al. Preanalytical aspects and sample quality assessment in metabolomics studies of human blood. Clin Chem 2013;59:833-45.

34. Gallagher D, Mhaolain AN, Crosby L, et al. Dependence and caregiver burden in Alzheimer's disease and mild cognitive impairment. Am J Alzheimers Dis Other Demen 2011;26:110-14.

35. Wattmo C, Wallin AK, Londos E, et al. Risk factors for nursing home placement in Alzheimer's disease: a longitudinal study of cognition, ADL, service utilization, and cholinesterase inhibitor treatment. Gerontologist 2011;51:17-27.

36. Jelic V, Winblad B. Alzheimer disease: donepezil and nursing home placement-benefits and costs. Nat Rev Neurol 2016:12:11-13.

37. Ross JS, Slodkowska EA, Symmans WF, et al. The HER-2 receptor and breast cancer: ten years of targeted anti-HER-2 therapy and personalized medicine. Oncologist 2009;14:320-68.

38. Testad I, Corbett A, Aarsland D, et al. The value of personalized psychosocial interventions to address behavioral and psychological symptoms in people with dementia living in care home settings: a systematic review. Int Psychogeriatr 2014;26:1083-98.

39. Bredesen DE, Amos EC, Canick J, et al. Reversal of cognitive decline in Alzheimer's disease. Aging (Albany NY) 2016;8:1250-8. 